\title{
The effect of home versus rehabilitation environment on activity levels of stroke survivors: Go home, sit less
}

Dawn B Simpson 1,2 , Monique Breslin1, Toby Cumming ${ }^{3}$, Seana L Gall ${ }^{1}$, Matthew Schmidt ${ }^{6}$, Coralie English ${ }^{4,5}$, and Michele L Callisaya ${ }^{1,2}$

${ }_{3}^{1}$ Menzies Institute of Medical Research, University of Tasmania, Hobart, Australia; ${ }^{2}$ Physiotherapy Department, Royal Hobart Hospital, Tasmanian Health Service - South, Hobart, Australia;

${ }_{5}^{3}$ Stroke Division, Florey Institute of Neurosciences and Mental Health, Melbourne, Australia; ${ }^{4}$ School of Health Sciences, University of Newcastle, Newcastle, Australia;
5 University of Newcastle Priority Research Centre for Stroke and Brain Injury, Hunter Medical Research Institute, Newcastle, Australia; ${ }^{5}$ School of Health Sciences, University of Tasmania, Hobart, Australia

Background: Prolonged sitting and low physical activity after stroke reduce function and increase cardiovascular risk. The rehabilitation environment may influence activity behaviours, though this remains unclear.

Objective: To examine 1) whether change in rehabilitation environment (hospital or home) and 2) what other factors, influence time sitting, upright and walking after stroke.

\section{Methods}

- Participants $(n=34)$ with stroke from $2 x$ inpatient rehabilitation units

- Observational study, 2 phases: final week inpatient rehabilitation; first week post discharge at home

Inclusion: admitted with stroke; mobile pre stroke; able to consent to participate; discharge to community

Exclusion: discharge to residential care

\section{Measures}

Activity measures:

- Sitting time - total daily (primary outcome)

- Upright time, walking time, steps/day

- activPAL3 monitor, worn 7 days (24 hours/day)

- 2 time points: hospital and home
Time 1 - Hospital

Activity final 7 days

Time 2 - Home Activity first 7 days
Characteristics (independent variable)

- Age, sex, stroke type, severity, carer present

Physical measures

- 10mw; 6MW; 5x STS test; Motor FIM scores

- Pain (VAS)

Psychological measures

- Hospital Anxiety \& depression scale

- Fatigue assessment scale

Cognition

- MoCA

\section{Data analysis}

- Descriptive statistics for participant characteristics

- Linear mixed model: activity as outcome, environment (hospital or home) as exposure

- Models adjusted for waking hours, age, sex

- Interaction terms created to determine factors that may modify activity with change in environment: fatigue, anxiety, depression, cognition, gait speed, gait capacity, FIM score, age, sex, stroke severity,

pain, carer

\section{Results}

\section{Participants}

- Mean age 68 [SD 13] years, 53\% male

- NIHSS admission score 7.2 [SD 5.4]

- Mean gait speed 0.98 [SD 0.58] m/s

- Mean 6 minute walk 280 [SD 165] m

- Mean MoCA score 22.1 [SD 5.6] points

Activity data

Mean waking hours:

- 13.1 [SD 1.5] h in hospital, 13.5 [SD 1.9] h at home Mean days activity data:

- Hospital $(\mathrm{n}=34)$ mean 5.6 [SD 1.9] days

- Home $(n=32)$ mean 6.7 [SD 0.6] days

Sitting and activity time with change in environment

- Significant differences in all activity measures between hospital and home environments (Table 1)

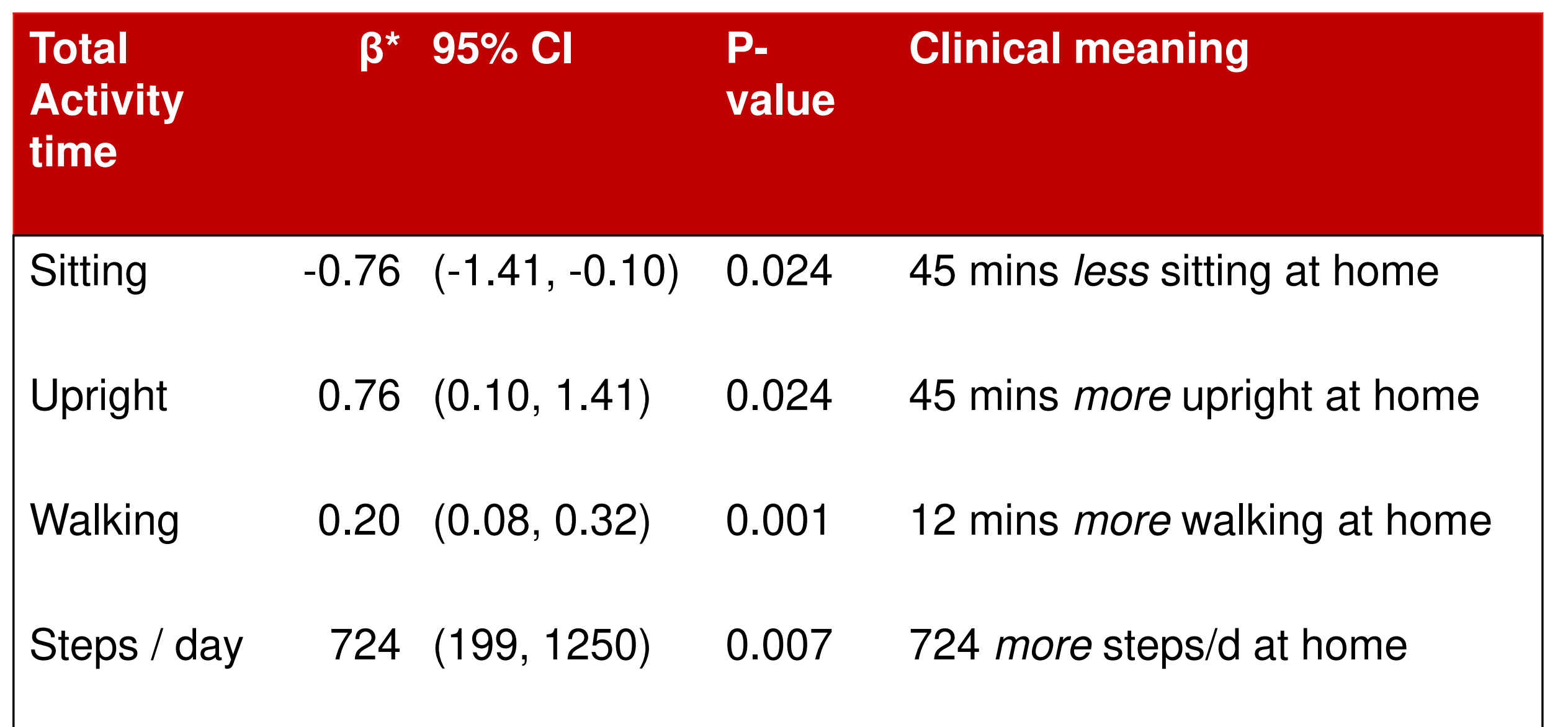

Table 1: Change in activity between hospital and home rehabilitation environments. $\beta^{\star}$ Adjusted mean difference from hospital to home (hours)

Activity modifiers

- Depression modified sitting and upright activity with change in environment $(p=0.03)$

- People with depression (figure 1) spent more time sitting and less time upright at home than in hospital

- No other covariates significantly modified activity - Hospital - Home

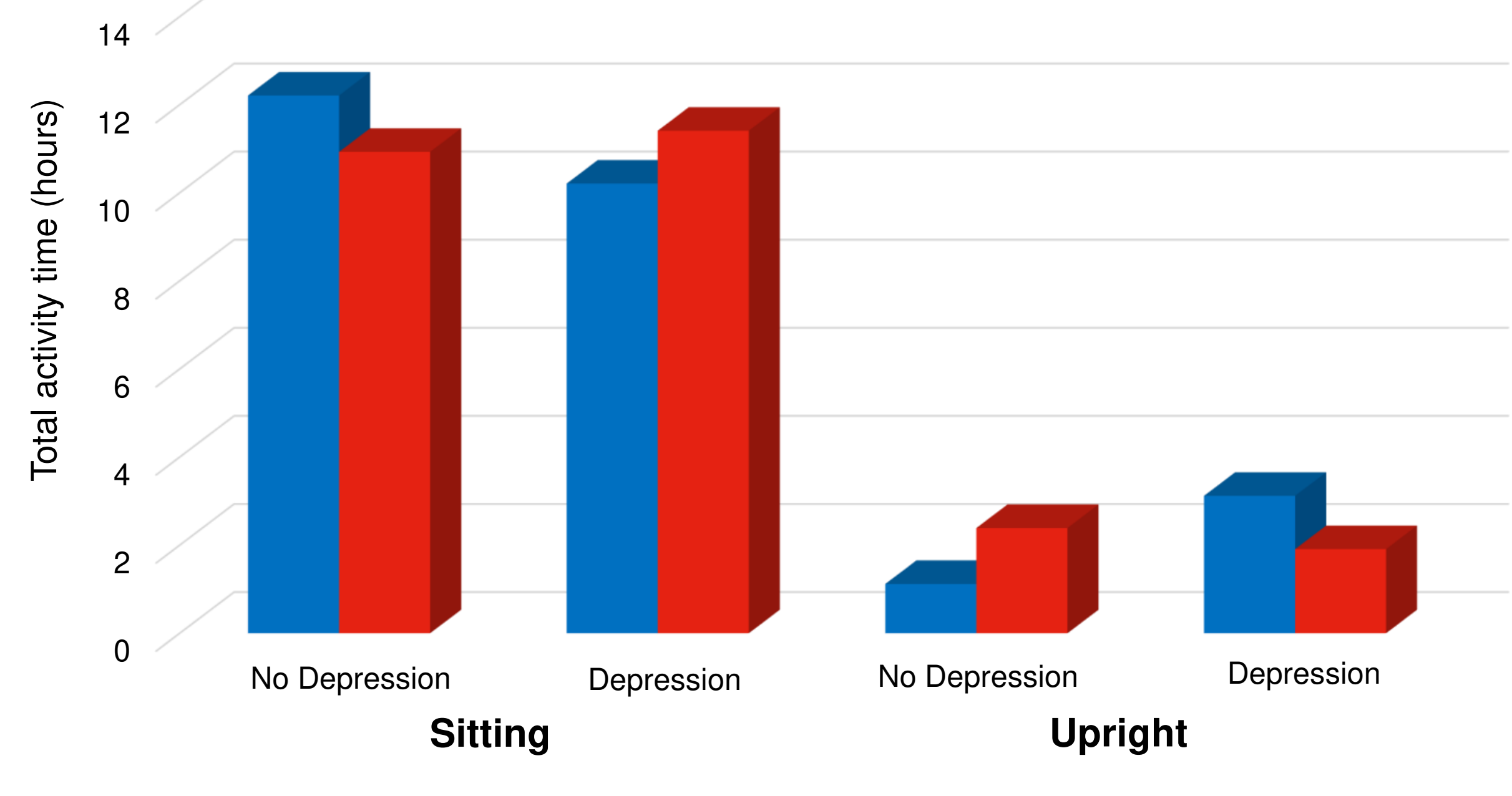

Figure 1: The effect of depression on sitting and upright time in the hospital and home environments
Contact Us

Menzies Institute for Medical Research For further information contact: Dawn Simpson Email: Dawn.Simpson@utas.edu.au @dsimpsonphysio www.menzies utas.edu.au
Acknowledgements

Our Participants

Funding:

Department of Geriatrics philanthropic award Tasmanian Health Service - South

NAHSSS Post Graduate Scholarship
- To further assess if functional recovery may influence change in activity with environment change, a secondary analysis (figure 2) was conducted to review sitting activity by day, across the observation period

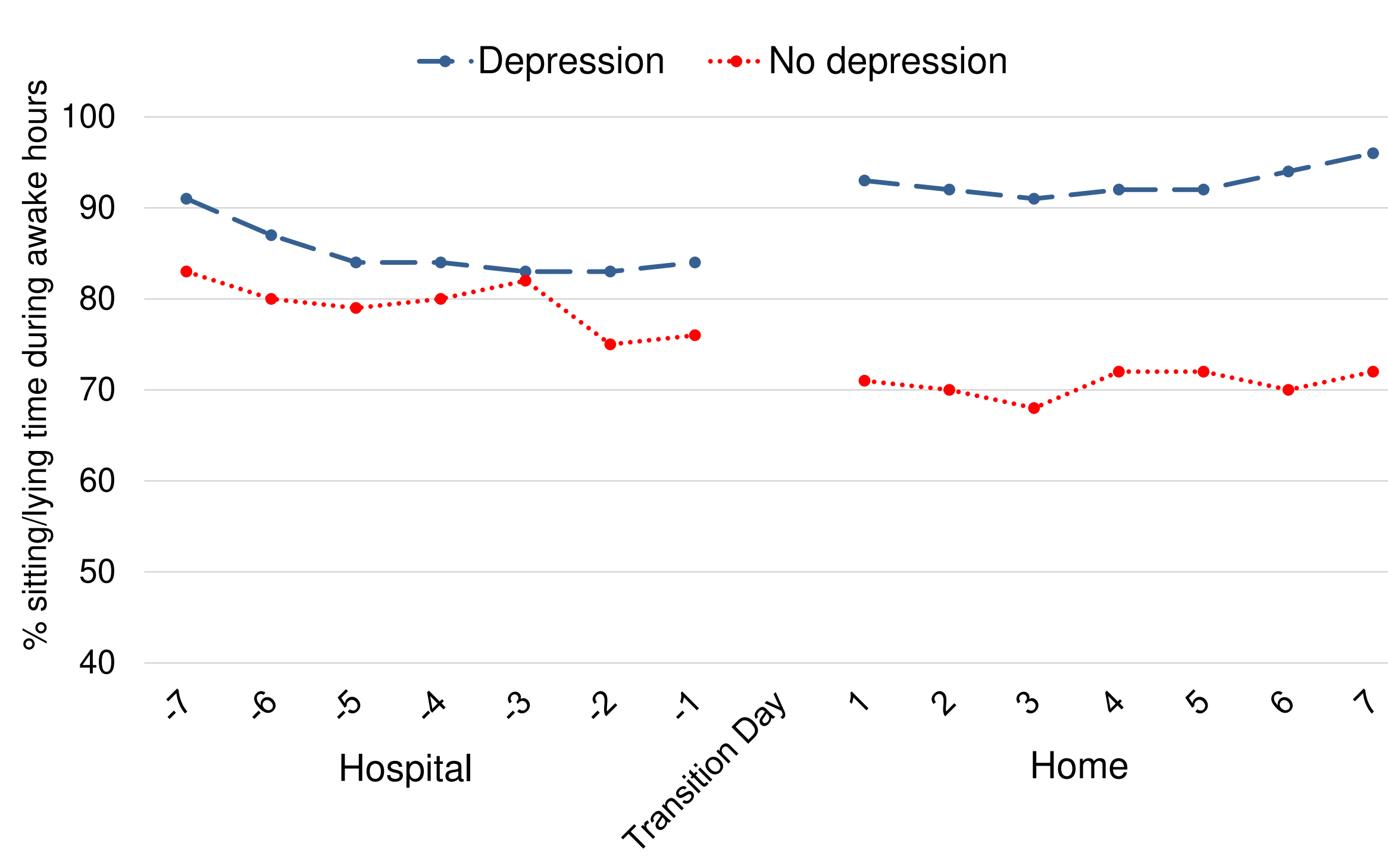

Figure 2: Mean percentage of waking day spent sitting across the 2 phases of observation, for participants with and without depression

\section{Conclusion}

- Change in environment from rehabilitation to home was associated with reduced sitting time, and greater time spent upright and walking.

\section{- Presence of depression} on discharge from hospital may negate the activity benefit of changing environment.
Department of Rehabilitation (THS-S)

Department of Geriatric Medicine (THS-S)

Physiotherapy Department (THS-S)

Dr Frank Nicklason - Department of Geriatrics (THS-S)

Dr Helen Castley - Department of Neurology (THS-S) 